\title{
Uso da galactose na vitrificação de sêmen ovino em palhetas
}

\section{Use of galactose to vitrify ram semen in straws}

\begin{abstract}
Márcio Calixto Matias ${ }^{1}$ (D) , Allan Rodolf Ribeiro Cézar ${ }^{1}$ (D) , Juliana Carla Cavalcanti Marques ${ }^{1}$ (D) , Fernanda Karla Ataide da Silva ${ }^{1}$ (D) , Vitória Nayreli Freire Gonçalves Sandes ${ }^{1}$ (D), Diogo Ribeiro Câmara ${ }^{1 *}$ (D)
\end{abstract}

${ }^{1}$ Universidade Federal de Alagoas (UFAL), Viçosa, AL, Brasil

${ }^{*}$ Correspondente: diogo@vicosa.ufal.br

Recebido em

28 de janeiro de 2021.

Aceito em

22 de abril de 2021.

Publicado

15 de junho de 2021.

www.revistas.ufg.br/vet Como citar - disponível no site, na página do artigo.

\section{Resumo}

A vitrificação de espermatozoides é uma técnica que apresenta grande potencial para criopreservação de material genético, e sua eficácia tem sido superior aos métodos convencionais em algumas espécies. No entanto, existem poucos estudos sobre sua eficiência com sêmen ejaculado de carneiros e o uso da galactose como crioprotetor extracelular durante a vitrificação. Objetivou-se com este estudo avaliar o efeito da galactose $(0,01$ M), associada ou não ao glicerol (3\% e 7\%), em meio comercial (Steridyl ${ }^{\circledR}$ - controle), na criopreservação de espermatozoides de carneiros pelo método de palhetas, comparando o método clássico de congelação e a vitrificação. Ejaculados de seis carneiros da raça Dorper em idade reprodutiva foram coletados com vagina artificial, aliquotados, diluídos individualmente $\left(100 \times 10^{6}\right.$ espermatozoides $\left./ \mathrm{mL}\right)$ nos meios testados, envasados em palhetas de 0,25 mL e submetidos à congelação clássica ou vitrificação. Foram analisadas a cinemática, morfologia, morfometria, viabilidade, integridade física e funcional da membrana espermática. A congelação clássica obteve melhores resultados de motilidade total e progressiva do que a vitrificação nos quatro extensores testados, uma vez que as amostras vitrificadas não apresentaram motilidade pósreaquecimento $(p<0,05)$. A adição de galactose ou glicerol ao meio comercial não trouxe efeito benéfico tanto para a vitrificação quanto congelação clássica.

Palavras-chave: Biotecnologia de sêmen; Congelamento ultrarrápido; Crioprotetores; Reprodução.

\begin{abstract}
Sperm vitrification is a technique with great potential for cryopreservation of genetic material, with superior effectiveness compared to conventional methods in some species. However, few studies have shown its efficiency with ejaculated sperm of rams and the use of galactose as an extracellular cryoprotectant during vitrification. This study aimed to evaluate the effect of galactose $(0.01 \mathrm{M})$, with or without glycerol addition (3\% and $7 \%$ ) in a commercial extender (Steridyl ${ }^{\circledR}$ - control) for ram sperm cryopreservation in straws, comparing the classic freezing method and
\end{abstract}


vitrification. Ejaculates from six breeding soundness Dorper rams were collected with an artificial vagina, aliquoted, individually diluted $\left(100 \times 10^{6} \mathrm{sperm} / \mathrm{mL}\right)$ on extenders tested, loaded into $0.25 \mathrm{~mL}$ straws, and subjected to a classic freezing method or vitrification. Sperm kinematics, morphology, morphometry, viability, and physical and functional integrity of the sperm membrane were evaluated. The classic freezing method resulted in higher total and progressive motility than vitrification, as no motility was detected in vitrified samples after rewarming $(p<0.05)$. The addition of galactose or glycerol to the commercial medium did not benefit both vitrification and the classic freezing method.

Keywords: Semen biotechnology; Ultra-rapid freezing; Cryoprotectants; Reproduction.

\section{Introdução}

A criopreservação de espermatozoides é uma ferramenta importante para conservação de genética animal, pois proporciona o armazenamento de material biológico viável por tempo indeterminado(1). Quando associada a biotécnicas como a inseminação artificial (IA), produção in vitro de embriões (PIV) e injeção intracitoplasmática de espermatozoides (ICSI), potencializa o ganho genético, refletindo no aumento da produtividade de rebanhos ${ }^{(2)}$.

Na espécie ovina, até o momento, os melhores resultados com a criopreservação espermática foram obtidos com o método de congelação convencional/clássica. No entanto, essa técnica ainda apresenta algumas limitações, sendo a formação de cristais de gelo intracelular a principal causa de danos aos espermatozoides durante o congelamento, provocando fragmentação no DNA e ruptura irreversível da membrana plasmática, reduzindo a motilidade dos espermatozoides após o descongelamento ${ }^{(3)}$. Novos métodos de criopreservação estão sendo desenvolvidos, na tentativa de obter resultados satisfatórios e visando contornar os problemas encontrados na congelação clássica, sendo a vitrificação uma das técnicas mais promissoras ${ }^{(4)}$.

A vitrificação de espermatozoides é uma técnica de criopreservação ultrarrápida e de baixo custo, podendo ser realizada a campo, pois não necessita de muitos equipamentos. É caracterizada por utilizar baixas concentrações de agentes crioprotetores ${ }^{(5)}$ e imersão direta do sêmen em nitrogênio líquido, provocando quedas de temperaturas que podem ser superiores a $10.000^{\circ} \mathrm{C} / \mathrm{min}^{(4)}$. Estas condições provocam a mudança do estado líquido para o estado amorfo da matéria, no qual o fluido que permanece no espaço intracelular adquire uma característica vítrea, impedindo a formação de cristais de gelo, e a ausência de estrutura atômica definida permite adequada dispersão da substância vítrea entre as organelas ${ }^{(6)}$. Esta técnica encontra-se bem estabelecida em humanos ${ }^{(7)}$, e experimentalmente apresenta bons resultados em jumentos ${ }^{(8)}$, equinos ${ }^{(9)}$, cães $^{(10)}$ e gatos $^{(6)}$.

Em ovinos, os melhores resultados obtidos após vitrificação são observados com sêmen coletado da cauda de epidídimo, enquanto para sêmen ejaculado os resultados 
são inferiores quando comparados à congelação clássica(11-13). No entanto, apenas a

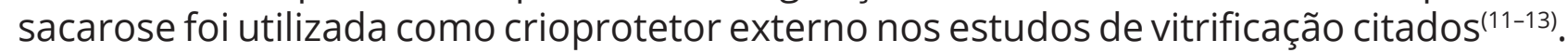
A utilização de outros carboidratos, como a galactose, pode promover melhores resultados, como observado durante a vitrificação de oócitos felinos ${ }^{(14)}$ e blastocistos equinos $^{(15)}$. Este monossacarídeo é capaz de inibir a recristalização do gelo durante o ciclo de congelação/descongelação, apresentando baixa citotoxicidade em temperaturas fisiológicas e capacidade de reduzir o estresse osmótico ${ }^{(16)}$. Já a adição da gema de ovo como crioprotetor nos extensores trouxe efeito benéfico para espermatozoides da espécie ovina ${ }^{(11,12)}$. Diante do exposto, objetivou-se, com este trabalho, avaliar o efeito da galactose, associada ou não ao glicerol, na criopreservação de espermatozoides de carneiros pelo método de palhetas, comparando o método clássico e a vitrificação.

\section{Material e métodos}

\section{Animais}

Este estudo foi autorizado pelo comitê de ética para uso de animais da Universidade Federal de Alagoas (54/2018). Ejaculados de nove animais da raça Dorper com idade entre 15 e 24 meses foram coletados com auxílio de vagina artificial, na cidade de Maceió, Alagoas. Os ejaculados foram avaliados de acordo com os critérios do Colégio Brasileiro de Reprodução Animal(17). Após realizado o exame, os ejaculados de seis reprodutores se encontravam dentro dos critérios mínimos estabelecidos para a criopreservação e foram utilizados para este estudo.

\section{Avaliação do sêmen}

Imediatamente após a coleta, os ejaculados foram submetidos às avaliações macroscópicas de volume, cor, odor e aspecto. Em seguida, foram efetuadas as análises microscópicas de turbilhonamento (0 - 5), motilidade progressiva (0 - 100\%) e o vigor espermático $(0-5)^{(17)}$. A concentração espermática foi determinada com auxílio de espectrofotometria, utilizando-se curva de calibração prévia $(r>0,95)$. Após diluição do ejaculado na proporção de 1:400 em solução tampão de gluteraldeido (citrato de sódio a 2,94\% e gluteraldeido a 2\%) a leitura da absorbância foi efetuada em comprimento de onda de 540 nM (V-1100, J.P. Selecta, Barcelona, Espanha).

Para avaliação da integridade física da membrana espermática, foi utilizado o corante supravital eosina/nigrosina (Minitube $\mathrm{GmbH}$, Tiefenbach, Alemanha) ${ }^{(10)}$. A integridade funcional da membrana espermática foi avaliada através do teste hiposmótico. Após diluição de $10 \mu \mathrm{L}$ de sêmen em $90 \mu \mathrm{L}$ de solução hiposmótica (7,35 g de citrato de sódio, 13,5 g de frutose, água destilada q.s.p. $1000 \mathrm{~mL}$ ), as amostras foram incubadas em banho-maria a $37^{\circ} \mathrm{C}$ (30 minutos), seguida da adição de $2 \mu \mathrm{L}$ de solução tampão de gluteraldeído a 5\%, para interromper a reação ${ }^{(18)}$. Uma alíquota de $10 \mu \mathrm{L}$ de cada amostra foi depositada em lâmina, sob lamínula, avaliando-se 200 espermatozoides em microscópio de contraste de fase (400×). O resultado foi expresso em porcentagem, após subtração do percentual de espermatozoides com patologia de cauda detectados durante o exame de morfologia espermática, de acordo com Oliveira et al.(19) 
A morfologia espermática foi avaliada através da técnica de câmara úmida, adicionando $10 \mu \mathrm{L}$ de sêmen a $100 \mu \mathrm{L}$ de solução tampão de gluteraldeído (5\%). Em seguida, uma alíquota de $10 \mu \mathrm{L}$ foi colocada em lâmina, sob lamínula, e levada ao microscópio de contraste de fase (400x), sendo avaliados 200 espermatozoides de acordo com o CBRA $^{(17)}$.

\section{Extensores para criopreservação}

Para avaliar a resistência individual à criopreservação, foram utilizados quatro extensores, tendo como base o extensor comercial Steridy ${ }^{\circledR}$ (Minitube, Alemanha): 1) Steridy ${ }^{\circledR}$ (Controle); 2 ) Steridy $\mid{ }^{\circledR}+0,01$ M de galactose; 3 ) Steridy ${ }^{\circledR}+0,01$ M de galactose $+3 \%$ de glicerol; 4) Steridy ${ }^{\circledR}+0,01 \mathrm{M}$ de galactose $+7 \%$ de glicerol.

\section{Criopreservação}

Após determinar a concentração espermática do ejaculado, o sêmen foi aliquotado nos quatro diferentes extensores, de forma a se obter uma concentração final de $100 \times 10^{6}$ espermatozoides móveis $/ \mathrm{mL}$. Em seguida, o sêmen foi envasado em palhetas de 0,25 $\mathrm{mL}$ e selado em seladora de palhetas MAXI30 (Neovet, Uberaba, Brasil). As amostras foram então submetidas à congelação clássica ou vitrificação.

\section{Congelação Clássica}

As palhetas foram acondicionadas em caixa isotérmica para transporte de sêmen (BotuFlex ${ }^{\circledR}$, Botupharma, Brasil), previamente refrigerada a $5{ }^{\circ} \mathrm{C}$, onde permaneceram por duas horas. Após a curva de refrigeração, foram transferidas para rampas $7 \mathrm{~cm}$ acima do nível do nitrogênio líquido e expostas ao vapor de nitrogênio (15 min) em caixa isotérmica, seguido de imersão em nitrogênio líquido(20). Após este processo, as palhetas foram acondicionadas em botijões criobiológicos $\left(-196^{\circ} \mathrm{C}\right)$ até as análises.

Vitrificação

Para vitrificação, as palhetas de $0,25 \mathrm{~mL}$ foram inseridas em palhetas de $0,50 \mathrm{~mL}$ e imersas diretamente no nitrogênio líquido(21). Em seguida, as palhetas foram acondicionadas em botijões criobiológicos $\left(-196^{\circ} \mathrm{C}\right)$ até as análises.

\section{Avaliações pós-criopreservação}

As amostras submetidas à congelação clássica foram descongeladas em banho-maria a $37^{\circ} \mathrm{C} / 30$ segundos, enquanto as amostras vitrificadas foram reaquecidas em banhomaria a $60^{\circ} \mathrm{C} / 5$ segundos ${ }^{(11)}$. Em seguida, o conteúdo das palhetas foi depositado em tubos de polipropileno a $37^{\circ} \mathrm{C}$, em banho-maria, procedendo-se as análises de integridade física e funcional da membrana espermática e morfologia espermática. Foram ainda realizadas as seguintes análises:

\section{Análise computadorizada - CASA}

Uma alíquota de $10 \mu \mathrm{L}$ de cada amostra foi diluída em $30 \mu \mathrm{L}$ de Steridy ${ }^{\circledR}$, homogeneizada e mantida em banho-maria por cinco minutos. Em seguida, uma alíquota de $2 \mu \mathrm{l}$ foi adicionada a uma câmara de contagem espermática (HC-B028C, Guangzou Electronics, China) e levada ao microscópio óptico de contrate de fase (100×). 
A cinemática dos espermatozoides foi avaliada utilizando o módulo de motilidade da extensão OpenCasa v1.0(22), com auxílio do Software ImageJ v1.52. As imagens foram capturadas a 30 quadros por segundo (FPS), através de uma câmera USB modelo OPT12 (Opticam Microscopy Technology), acoplado ao microscópio de contraste de fase FWL 3500TFL (Feldmann Wild Leitz, Manaus, Brasil). De cada amostra de sêmen, foram capturados cinco vídeos com duração de dois segundos, sendo avaliados no mínimo 500 espermatozoides por amostra.

\section{Viabilidade espermática através de sonda fluorescente LIVE/DEAD}

Para esta análise foi utilizado o kit comercial Live/Dead (Invitrogen, EUA). Dez microlitros de sêmen foram homogeneizados com $1 \mu \mathrm{L}$ de SYBR14 (1 mM) e $1 \mu \mathrm{L}$ de iodeto de propídeo $(2,4 \mathrm{mM})$ em um tubo de polipropileno. Após incubação por dez minutos em banho-maria $\left(37^{\circ} \mathrm{C}\right)$, foi adicionado $1 \mu \mathrm{l}$ de solução tampão de gluteraldeido (2\%) para fixar os espermatozoides. Uma alíquota de $10 \mu \mathrm{L}$ foi adicionado em lâmina, sob lamínula, e levada ao microscópio de fluorescência, no comprimento de onda de 450 $490 \mathrm{~nm}$. Após a captura das imagens, foram avaliados 200 espermatozoides por amostra com auxílio do módulo de viabilidade do OpenCasa v1.0, com aumento de 200x.

\section{Morfometria espermática}

Para a morfometria da cabeça dos espermatozoides foi utilizada a mesma lâmina confeccionada para viabilidade (SYBR14 e iodeto de propídeo). As imagens capturadas em microscopia de fluorescência foram avaliadas através do módulo de morfometria do OpenCasa v1.0, sendo analisados 200 espermatozoides por amostra, com aumento de $400 x^{(22) .}$

\section{Análise estatística}

As variáveis utilizadas para comparar os parâmetros espermáticos foram: as duas técnicas de criopreservação (congelação clássica e vitrificação) e os quatro diferentes extensores. Os resultados obtidos de cada ejaculado, em cada um dos tratamentos, foram agrupados, sendo expressos na forma de média \pm erro padrão. Para avaliar a normalidade dos dados foi usado o teste de Kolmogorov-Smirnov. As médias foram comparadas utilizando-se análise de variância (One-Way ANOVA), seguido do teste de Tukey. Todos os dados foram analisados através do software RStudio v1.4 e as diferenças foram consideradas significantes quando $p<0,05$.

\section{Resultados}

Na congelação clássica, a adição de galactose a 0,01 $\mathrm{M}$ isoladamente não diferiu do grupo controle (Steridyl ${ }^{\circledR}$ ) em nenhum dos parâmetros estudados (Tabelas 1 e 2). A adição do glicerol a 3\% associado à galactose reduziu apenas a viabilidade espermática $(p<0,05)$. No entanto, quando foi adicionado glicerol na concentração de $7 \%$, observouse redução significativa da motilidade total e progressiva, da viabilidade, da integridade física e funcional da membrana. Quando utilizada a técnica de vitrificação espermática, a adição de galactose $0,01 \mathrm{M}$, isoladamente ou associada ao glicerol nas concentrações de $3 \%$ ou $7 \%$, não apresentou diferença $(p>0,05)$ em nenhum dos parâmetros avaliados 
em relação ao diluente controle, (Tabelas 1 e 2). Todavia, ao comparar as técnicas de criopreservação, a vitrificação espermática apresentou resultados inferiores à congelação clássica $(p<0,05)$, pois não apresentou motilidade pós-reaquecimento em nenhum dos extensores, bem como menores valores de viabilidade, integridade física e funcional da membrana (Tabela 1)

A maioria dos extensores utilizados não influenciou os parâmetros morfométricos da cabeça espermática, independentemente da técnica de criopreservação, com exceção do extensor 4 (Steridy ${ }^{\circledR}+0,01 \mathrm{M}$ galactose $+7 \%$ glicerol), que apresentou valores menores $(p<0,05)$ de área e largura da cabeça espermática na vitrificação quando comparada à congelação clássica (Tabela 1).

Dentro da mesma técnica de criopreservação (vitrificação ou congelação clássica), a adição de galactose $0,01 \mathrm{M}$ e glicerol a $3 \%$ ou $7 \%$ ao meio Steridy ${ }^{\circledR}$ não influenciou em nenhum dos parâmetros morfológicos avaliados $(p>0,05)$. No entanto, ao se comparar as diferentes técnicas de criopreservação, foi observado que a vitrificação espermática resultou em maior porcentagem de cauda fortemente enrolada e menor percentual de células reativas ao teste hiposmótico em todos os extensores, quando comparada à congelação clássica $(p<0,05)$. Além disso, a utilização de extensores adicionados de galactose, com ou sem glicerol a 3\% e 7\%, aumentou significativamente o percentual de defeitos maiores. Foram observadas ainda algumas diferenças entre a vitrificação e a congelação clássica no percentual de células com gota proximal, cauda enrolada na cabeça e cauda dobrada ou enrolada, mas sem uma clara distinção entre os diluidores e sempre numa pequena proporção das células avaliadas (Tabela 2). 
Uso da galactose na vitrificação de sêmen ovino em palhetas

Matias MC et al.

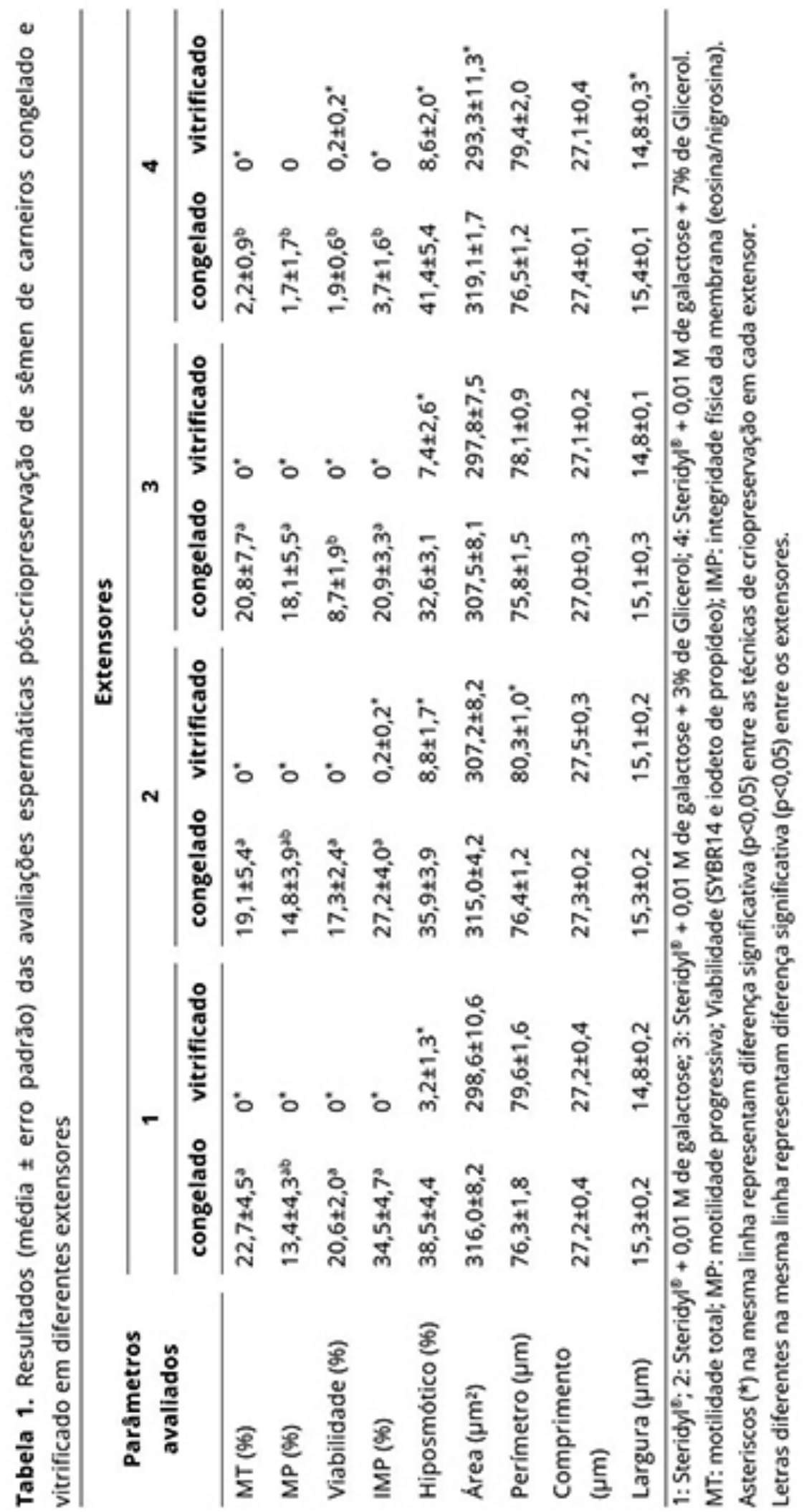




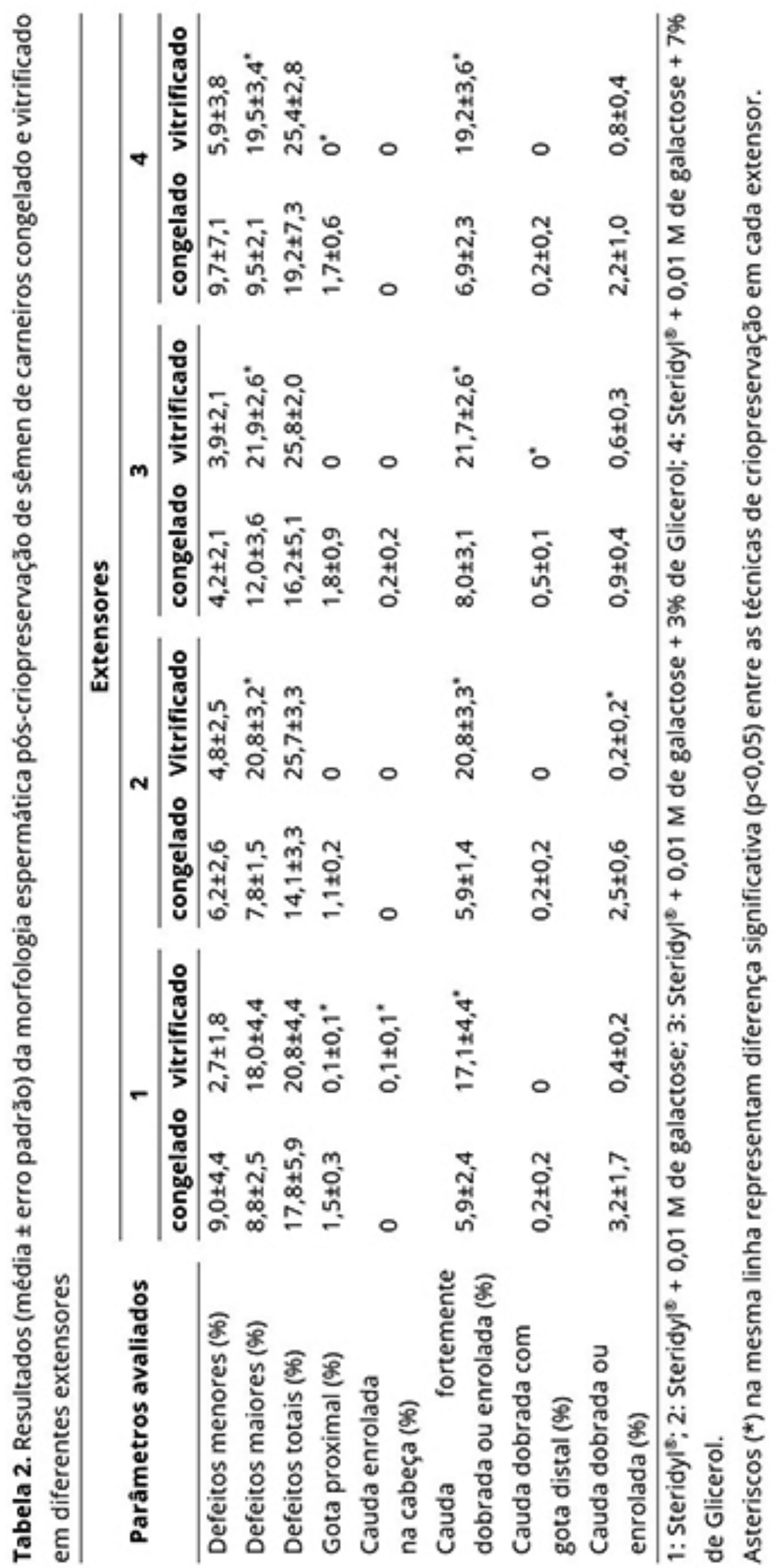




\section{Discussão}

No presente estudo, a galactose não foi capaz de melhorar os resultados observados após a vitrificação de espermatozoides ejaculados de carneiros, ao contrário do relatado durante a vitrificação de oócitos felinos ${ }^{(14)}$ e blastocistos equinos ${ }^{(15)}$. Além disso, os resultados observados foram inferiores aos reportados para o uso da mesma concentração de sacarose com sêmen de ovinos Muflon ${ }^{(11)}$. O extensor Steridyl ${ }^{\circledR}$ foi escolhido neste experimento por ser específico para ruminantes e apresentar gema de ovo em sua composição, tendo sido relatado efeito benéfico da gema de ovo em extensores utilizados para vitrificação de sêmen ovino ${ }^{(23)}$. Todavia, após o reaquecimento, as amostras vitrificadas não apresentaram motilidade quando analisadas pelo CASA e tiveram baixos valores de viabilidade, integridade física e funcional de membrana, fator crucial para o sucesso da técnica. No entanto, em algumas amostras de sêmen vitrificadas, espermatozoides microcefálicos apresentaram movimento, mas devido à área da cabeça as células não foram registradas pelo software CASA. Essa observação vai ao encontro da afirmação de Isachenko et al.(24), que relataram maior crioresistência à vitrificação de espermatozoides que apresentam como característica espécieespecífica um menor tamanho de cabeça.

No presente estudo, não foi observado efeito benéfico da adição da galactose na qualidade espermática pós-descongelação ou reaquecimento. Diferente da vitrificação de oócitos e embriões ${ }^{(4)}$, a vitrificação de espermatozoides utiliza baixa concentração de agentes crioprotetores, ou até mesmo a ausência deles ${ }^{(7)}$, objetivando menor estresse osmótico e danos aos espermatozoides ${ }^{(24)}$. Assim, optou-se por utilizar uma baixa concentração de galactose, pois os espermatozoides de carneiros são potencialmente sensíveis a altas concentrações de carboidratos nos extensores de vitrificação, uma vez que os melhores resultados de motilidade pós-reaquecimento foram obtidos quando o carboidrato testado (sacarose) foi utilizado em baixas concentrações ${ }^{(11,25)}$.

Para avaliar o efeito da associação com crioprotetor penetrante, foi adicionado glicerol nas concentrações de 3\% e 7\% ao extensor contendo 0,01 M de galactose. Todavia, não foram observados efeitos positivos, pois as amostras apresentaram baixa viabilidade e ausência de motilidade após o reaquecimento em ambas as concentrações de glicerol testadas. Este resultado foi semelhante ao encontrado por Jiménez-Rabadán et al.(25) e Pradiee et al.(11), que demonstraram efeitos deletérios do glicerol em amostras vitrificadas. Vale ressaltar que na congelação clássica, a adição de 3\% de glicerol não foi deletéria, apresentando resultado similar ao Steridyl ${ }^{\circledR}$ e Steridyl ${ }^{\circledR}$ com adição de 0,01 M de galactose. Contudo, na concentração de 7\%, o glicerol foi prejudicial nos parâmetros de motilidade total, progressiva, viabilidade, integridade física e funcional da membrana. Apesar dessa toxicidade ser esperada, uma vez que o extensor comercial já apresenta glicerol em sua composição e que concentrações de glicerol superiores a 6\% podem ser tóxicas para espermatozoides ovinos(26), não se sabia o efeito da associação entre a galactose e maiores concentrações de glicerol durante a vitrificação, mantendo-se o mesmo extensor também para a congelação clássica, de forma a não prejudicar o desenho experimental. 
Ao avaliar a integridade funcional da membrana pelo teste hiposmóstico, não houve diferença significativa entre os extensores para ambas as técnicas de criopreservação, sendo que esse resultado pode ser atribuído à adição de galactose a 0,01 M, que tem ação osmoprotetora, característica dos crioprotetores extracelulares já citados ${ }^{(14)}$. Todavia, deve-se destacar que o teste hiposmótico simples não é capaz de detectar subpopulações espermáticas com alta resistência osmótica e que retornam ao seu volume inicial após o transporte ativo de água e íons através da membrana (sendo classificados como não reativos ao teste); células com resistência osmótica muito baixa, que se rompem durante o teste (consequentemente, não quantificadas); ou mesmo células não viáveis desde o início do teste, sendo incapazes de responder ao estímulo hiposmótico(27).

Morfologicamente não houve diferença significativa entre os extensores utilizados dentro da mesma técnica de criopreservação. Entretanto, ao comparar as técnicas de criopreservação, foi observado que a vitrificação espermática resultou em maior porcentagem de cauda fortemente enrolada em todos os extensores quando comparada com a congelação clássica. Esse resultado é semelhante ao encontrado por Arando et al. (28), que ao criopreservarem espermatozoides de carneiros da raça Merino observaram que a vitrificação causa mais defeitos de cauda em relação à congelação clássica, provavelmente devido a alterações de flagelo observadas em microscopia eletrônica de transmissão, como a presença de vesículas e áreas de dilatação sob a membrana plasmática, além de alterações na forma e dimensão das mitocôndrias dos espermatozoides. Acredita-se que esses danos ocorrem no momento do reaquecimento, pois, segundo Curry e Watson ${ }^{(29)}$, os espermatozoides ovinos são mais sensíveis que os humanos ao saírem de um meio hiperosmótico para um meio isosmótico, característica que pode se refletir na maior qualidade espermática observada após a vitrificação de espermatozoides humanos, quando comparada à espécie ovina.

A resistência osmótica dos espermatozoides é uma variável importante para a qualidade espermática pós-vitrificação, uma vez que os melhores resultados já publicados de vitrificação de sêmen ovino foram observados em espermatozoides obtidos da cauda do epidídimo(12), quando não houve diferença significativa entre a vitrificação e congelação clássica nos principais parâmetros avaliados, como a motilidade total, morfologia e integridade física de membrana. A maior crioresistência à vitrificação dos espermatozoides epididimários em relação aos espermatozoides ejaculados foi confirmada por Martínez-Fresneda et al.(13), evidenciando em seu estudo que os espermatozoides, ao terem contato com o plasma seminal, iniciam um processo semelhante à capacitação espermática, com aumento da fosforilação da tirosina, causando redução da resistência osmótica e diminuindo sua crioresistência.

A morfometria é um dos parâmetros que avaliam o efeito físico dimensional que os extensores e métodos de criopreservação exercem sobre os espermatozoides. No nosso estudo, não houve diferença significativa entre nenhum dos extensores dentro de cada uma das técnicas de criopreservação utilizadas. No entanto, ao comparar as técnicas de criopreservação, houve uma redução na área e largura dos espermatozoides vitrificados com o extensor que contêm $7 \%$ de glicerol. Essa redução pode ser consequência do alto 
percentual de espermatozoides com perda de integridade estrutural e funcional da membrana, associada a uma maior osmolaridade do meio, diminuindo a reidratação no pequeno percentual de células com membrana funcional pós-reaquecimento e culminando com uma redução das dimensões da cabeça espermática, como observado por Arando et al. ${ }^{(28)}$

\section{Conclusões}

Concluiu-se que a adição de galactose ao meio Steridyl ${ }^{\circledR}$, associada ou não ao glicerol, é ineficaz para a vitrificação de espermatozoides ejaculados de carneiros em palhetas, além de não melhorar os resultados pós-descongelação quando os espermatozoides foram criopreservados pelo método clássico.

\section{Agradecimentos}

Os autores agradecem à CAPES/UFAL/FAPEAL (Código 001) pela bolsa de estudos. À Minitube do Brasil, pelo apoio para execução do experimento.

\section{Conflito de interesses}

Os autores declaram não haver conflito de interesses.

\section{Referências}

1. Pegg DE. The history and principles of cryopreservation. Seminars in Reproductive Medicine. 2002;20(1):5-13. https://doi.org/10.1055/s-2002-23515.

2. Rumpf R. Avanços metodológicos na produção in vitro de embriões. Revista Brasileira de Zootecnia. 2007;36(suppl.0):229-233. https://doi.org/10.1590/S1516-35982007001000021.

3. Bittencourt RF, Oba E, Ribeiro Filho AL, Chalhoub M, Azevedo HC, Bicudo SD. Avanços na criopreservação do sêmen ovino I: diluidores e crioprotetores. Ciência Animal Brasileira. 2013;14(4):522-536. https://doi. org/10.5216/cab.v14l4.22964.

4. Carvalho AA, Faustino LR, Figueiredo JR, Rodrigues APR, Costa APR. Vitrificação: uma alternativa para a preservação de embriões e material genético de fêmeas mamíferas em criobancos. Acta Veterinaria Brasilica. 2011;5(3):236-248. Disponível em: https://periodicos.ufersa.edu.br/index.php/acta/article/ view/2321.

5. Matias MC, Melo MMS, Mendonça MSB, Sousa CWC, Costa Neto LF, Câmara DR. Vitrificação de espermatozoides em pequenos ruminantes. Revista Brasileira de Reprodução Animal. 2019;43(2):105110. http://cbra.org.br/portal/downloads/publicacoes/rbra/v43/n2/p105-110\%20(RB776).pdf.

6. Vizuete G, Jiménez E, Agüera El, Pérez-Marín CC. Impact of ultra-rapid freezing on the motility, morphology, viability and acrosome integrity of epididymal cat sperm diluted in sucrose-based extenders. Reproduction in Domestic Animals. 2014;49(1):e5-8. https://doi.org/10.1111/rda.12253.

7. Slabbert M, du Plessis SS, Huyser C. Large volume cryoprotectant-free vitrification: an alternative to 
Uso da galactose na vitrificação de sêmen ovino em palhetas

Matias MC et al.

conventional cryopreservation for human spermatozoa. Andrologia. 2015;47(5):594-599. https://doi. org/10.1111/and.12307.

8. Diaz-Jimenez M, Dorado J, Ortiz I, Consuegra C, Pereira B, Gonzalez-De Cara CA, Aguilera R, Mari G, Mislei B, Love CC, Hidalgo, M. Cryopreservation of donkey sperm using non-permeable cryoprotectants. Animal Reproduction Science. 2018; 189:103-109. https://doi.org/10.1016/j.anireprosci.2017.12.013.

9. Hidalgo M, Consuegra C, Dorado J, Diaz-Jimenez M, Ortiz I, Pereira B, Sanchez R, Crespo F. Concentrations of non-permeable cryoprotectants and equilibration temperatures are key factors for stallion sperm vitrification success. Animal Reproduction Science. 2018;196:91-98. https://doi.org/10.1016/j. anireprosci.2018.06.022.

10. Pipan MZ, Casal ML, Šterbenc N, Klun IV, Mrkun J. Vitrification using soy lecithin and sucrose: a new way to store the sperm for the preservation of canine reproductive function. Animals. 2020;10(4):653. https://doi.org/10.3390/ani10040653.

11. Pradiee J, Esteso MC, Castaño C, Toledano-Díaz A, Lopez-Sebastián A, Guerra R, Santiago-Moreno, J. Conventional slow freezing cryopreserves mouflon spermatozoa better than vitrification. Andrologia. 2017;49(3):e12629. https://doi.org/10.1111/and.12629.

12. Bóveda $P$, Esteso MC, Castaño C, Toledano-Díaz A, López-Sebastián A, Muñiz A, Prieto P, Mejía O, Ungerfeld R, Santiago-Moreno J. Slow and ultra-rapid freezing protocols for cryopreserving mouflon (Ovis musimon) and fallow deer (Dama dama) epididymal sperm. Animal Reproduction Science. 2018;192:193199. https://doi.org/10.1016/j.anireprosci.2018.03.010.

13. Martínez-Fresneda L, Castaño C, Bóveda P, Tesfaye D, Schellander K, Santiago-Moreno J, García-Vázquez FA. Epididymal and ejaculated sperm differ on their response to the cryopreservation and capacitation processes in mouflon (Ovis musimon). Scientific Reports. 2019;9:15659. https://doi.org/10.1038/s41598019-52057-0.

14. Herrick JR, Wang C, Machaty Z. The effects of permeating cryoprotectants on intracellular free-calcium concentrations and developmental potential of in vitro-matured feline oocytes. Reproduction, Fertility and Development. 2016;28(5):599-607. https://doi.org/10.1071/rd14233.

15. Hinrichs K, Choi Y-H. Micromanipulation of equine blastocysts to allow vitrification. Reproduction, Fertility and Development. 2016;28(8):1092-1096. https://doi.org/10.1071/rd15389.

16. Chaytor JL, Tokarew JM, Wu LK, Leclère M, Tam RY, Capicciotti CJ, Guolla L, von Moos E, Findlay CS, Allan DS, Ben RN. Inhibiting ice recrystallization and optimization of cell viability after cryopreservation. Glycobiology. 2012; 22(1):123-33. https://doi.org/10.1093/glycob/cwr115.

17. Colégio Brasileiro de Reprodução AnimaL. Manual para exame andrológico e avaliação de sêmen animal. 3a. Belo Horizonte: CBRA; 2013. 104 p.

18. Aisen EG, Medina VH, Venturino A. Cryopreservation and post-thawed fertility of ram semen frozen in different trehalose concentrations. Theriogenology. 2002; 57(7):1801-1808. https://doi.org/10.1016/ s0093-691X(02)00653-2.

19. Oliveira IRS, Alves HM, Castelo TS, Bezerra FSB, Bezerra ACDS, Silva AR. Correlações entre o teste hiposmótico e a avaliação clássica do sêmen de caprinos. Ciência Animal Brasileira. 2013;14(2):216-221. https://doi.org/10.5216/cab.v14i2.12921.

20. Masoud R, Sharafi M, Shahnenh AZ, Towhidi A, Kohram H, Zhandi M, Esmaeili V, Shahverdi A. Effect of dietary fish oil supplementation on ram semen freeze ability and fertility using soybean lecithin- and egg-yolk-based extenders. Theriogenology. 2016; 86(6):1583-1588. http:// dx.doi.org/10.1016/j.theriogenology.2016.05.018. 
21. Diaz-Jimenez M, Dorado J, Pereira B, Ortiz I, Consuegra C, Bottrel M, Ortiz E, Hidalgo M. Vitrification in straws conserves motility features better than spheres in donkey sperm. Reproduction in Domestic Animals. 2018;53(Suppl.2):56-58. https://doi.org/10.1111/rda.13256.

22. Alquézar-Baeta C, Gimeno-Martos S, Miguel-Jiménez S, Santolaria P, Yániz J, Palacín I, Casao A, Cebrián-Pérez JA, Muiño-Blanco T, Pérez-Pé R. OpenCASA: A new open-source and scalable tool for sperm quality analysis. PLOS Computational Biology. 2019;15(1):e1006691. https://doi. org/10.1371/journal.pcbi.1006691.

23. Arando A, Gonzalez A, Delgado JV, Arrebola FA, Perez-Marín CC. Storage temperature and sucrose concentrations affect ram sperm quality after vitrification. Animal Reproduction Science. 2017;181:175-185. https://doi.org/10.1016/j.anireprosci.2017.04.008.

24. Isachenko E, Isachenko V, Katkov II, Dessole S, Nawroth F. Vitrification of mammalian spermatozoa in the absence of cryoprotectants: from past practical difficulties to present success. Reproductive BioMedicine Online. 2003;6(2):191-200. https://doi.org/10.1016/s1472$\underline{6483(10) 61710-5 .}$.

25. Jiménez-Rabadán P, García-Álvarez O, Vidal A, Maroto-Morales A, Iniesta-Cuerda M, Ramón M, del Olmo E, Fernández-Santos R, Garde JJ, Soler AJ. Effects of vitrification on ram spermatozoa using free-egg yolk extenders. Cryobiology. 2015;71(1):85-90. https://doi.org/10.1016/j. cryobiol.2015.05.004.

26. Fiser PS, Fairfull RW. The effect of glycerol concentration and cooling velocity on cryosurvival of ram spermatozoa frozen in straws. Cryobiology. 1984;21(5):542-551. https:// doi.org/10.1016/0011-2240(84)90053-1.

27. Petrunkina AM, Waberski D, Günzel-Apel AR, Töpfer-Petersen E. Determinants of sperm quality and fertility in domestic species. Reproduction. 2007;134(1):3-17. https://doi. org/10.1530/rep-07-0046.

28. Arando A, Delgado JV, Arrebola FA, León JM, Alcalá CJ, Pérez-Marín CC. Vitrification induces critical subcellular damages in ram spermatozoa. Cryobiology. 2019;87:52-59. https://doi. org/10.1016/j.cryobiol.2019.02.005.

29. Curry MR, Watson PF. Osmotic effects on ram and human sperm membranes in relation to thawing injury. Cryobiology. 1994;31(1):39-46. https://doi.org/10.1006/cryo.1994.1005. 